\title{
Necrotizing Abdominal Fasciitis in Untreated Diabetes Mellitus
}

\author{
Laia Torrent-Jansà, Estela Membrilla-Fernández,, Marta Climent-Agustín,, Claudio Silva-Vergara, \\ Annabel Martínez-Sola, Sandra Toro-Araunabeña,' $\mathrm{M}^{a}$ José Pons-Fraguero, \\ Felip N. Isbert-Trullàs,' Juan Guzman-Ahumada,' Susana López-Fernández? \\ Luis Grande-Posa, and Juan Sancho-Insenser ${ }^{1}$
}

\begin{abstract}
Background: Necrotizing fasciitis (NF) is rare and can be fatal unless a prompt diagnosis and aggressive multidisciplinary treatment are performed. The present case aims to show how an innocent omphalitis, treated initially with topical antibiotic cream, can evolve to a potentially life-threatening condition such as NF of the entire abdominal wall. It illustrates a surgical and antibiotic management that not only saves patients lives, but also enables the repair of the severe tissue lesions.

Case Presentation: We present the case of a 44-year-old Caucasian female, diagnosed with diabetes mellitus 30 years ago that had never been treated. She came to the emergency department with a glycemia of $470 \mathrm{mg} / \mathrm{dL}$ and a $3-\mathrm{cm}$ diameter peri-umbilical necrotic scar. The initial blood investigation revealed serum glucose concentration of $559 \mathrm{mg} / \mathrm{dL}$, elevated acute phase reactants, and a metabolic acidosis. The computed tomography images were compatible with a necrotizing abdominal wall infection. A broad-spectrum antibiotic agent was prescribed empirically and urgent surgical debridement was performed. Negative pressure wound therapy (NPWT) was applied as well as several re-debridements. Polymicrobial flora grew on the wound exudate cultures so the spectrum could be changed according to the culture sensitivity of the microbial isolates and the clinical evolution of the patient. After 62 days of treatment, the wound cultures were negative and the abdominal wall could be reconstructed by means of a direct closure by abdominoplasty. In parallel, a strict glucose control was established as well as an educational intervention in order to improve long-term glycemic control. The clinical condition of the patient improved and she was discharged after 75 days of hospital stay and showed correct glycemia at outpatient visits. Conclusion: Necrotizing fasciitis of the entire anterior abdominal wall are unusual and have a poor prognosis if misdiagnosed at the initial stage as well as if indequate management and early surgical treatment are not performed. Initial broad-spectrum antibiotic agents as well as an initial aggressive surgical debridement are mandatory with further re-debridements as long as needed. Diabetes mellitus is a main predisposing factor to take into consideration.
\end{abstract}

Keywords: abdominal wall infection; diabetes mellitus; hyperglycemia; necrotizing fasciitis

$\mathbf{N}$ ECROTIZING FASCIITIS (NF) is a widespread necrosis of the subcutaneous tissue and fasciae. It is rare and can be fatal with a rapid and destructive clinical course. It is one of the most life-threatening surgical emergencies requiring a prompt diagnosis and aggressive multi-disciplinary treat- ment. An early recognition of its clinical features is mandatory because its diagnosis may not be apparent initially [1]. The clinician must be aware that a trivial skin lesion can be the starting point, such an insect bite, an abrasion or boil, presented as a cellulitis, which can advance rapidly. A systemic

\footnotetext{
${ }^{1}$ Emergency Unit, Digestive and General Surgery Department, ${ }^{2}$ Plastic Surgery Department, Hospital del Mar, Barcelona, Spain.

(C) Laia Torrent-Jansà et al. 2017; Published by Mary Ann Liebert, Inc. This Open Access article is distributed under the terms of the Creative Commons License (http://creativecommons.org/licenses/by/4.0), which permits unrestricted use, distribution, and reproduction in any medium, provided the original work is properly credited.
} 
toxicity may appear as well as more ominous skin signs with its progression. A broad erythematous tract may appear at the follow-up skin examination, as well as edema, cutaneous discoloration, anesthesia and gangrene with gas in the soft tissues. In practice, clinical judgment is the most important element in diagnosis and computed tomography (CT) or magnetic resonance imaging (MRI) may delay definitive diagnosis and treatment. Computed tomography and MRI may show edema extending along the fascial plane, although the sensitivity and specificity of these imaging studies are not well defined [2].

Surgical referral should be made as early as possible in order to perform tissue debridement and start a broad-spectrum antibiotic cover, both measures being essential to minimize morbidity and mortality rates [3]. An aggressive surgical intervention is essential as long as it is needed. The fascia appears to be swollen and gray in colord, with broad areas of necrosis at direct visual examination during surgical examination. Even if there is an important liquid exudate, no true pus is usually obtained. Most patients should return to the operating room 24-36 hours after the first debridement and daily thereafter until the surgical team finds no further need for debridement.

The causative agent is mainly a synergic polymicrobial infection in which bacteria produce virulent toxins. Numerous different aerobic and anaerobic organisms can be obtained, the source of which are the bowel or genitourinary flora. Regarding the monomicrobial infections, the usual pathogens are Streptococcus pyogenes, Staphylococcus aureus, Vibrio vulnificus, Aeromonas hydrophila, and anaerobic streptococci [4].

Most infections are acquired in the community, mainly in the lower extremities. However, nearly the half of patients with NF caused by Streptococcus pyogenes have no portal of entry but develop deep infection at the exact site of non-penetrating trauma such as a bruise or muscle strain. Often it is possible to detect a pre-disposing condition such as diabetes mellitus, arteriosclerotic vascular disease, and venous stasis with edema or vascular insufficiency, ulcer, or injection drug use. Also women who have recently delivered a child are at risk of NF [4].

Broad-spectrum antibiotic combinations against gramnegative, gram-positive bacilli, and anaerobes should be used as empirical treatment. Among the many choices are vancomycin, linezolid, or daptomycin combined with one of the following: either piperacillin-tazobactam or a carbapenem or ceftriaxone plus metronidazole, or a fluoroquinolone plus metronidazole [5,6]. Once the microbial etiology has been determined, the antibiotic coverage should be modified appropriately. The spectrum can be changed according to the culture sensitivity of the microbial isolates and the clinical evolution of the patient.

Some clinical scoring systems were created or applied to NF in order to reach a more rapid and scientific methods of diagnosis. The Laboratory Risk Indicator for Necrotizing Fasciitis (LRINEC) scoring system is an example as well as the Acute Physiology and Chronic Health Evaluation (APACHE II) scoring sysem [7,9]. Here we describe a case starting as innocent omphalitis treated initially with topical antibiotic cream and evolving to a life-threatening condition such as NF).

\section{Case Presentation}

We present the case of a 44-year-old Caucasian female, without allergies, who is a heavy cigarette smoker and who had been diagnosed with diabetes mellitus 30 years prior that

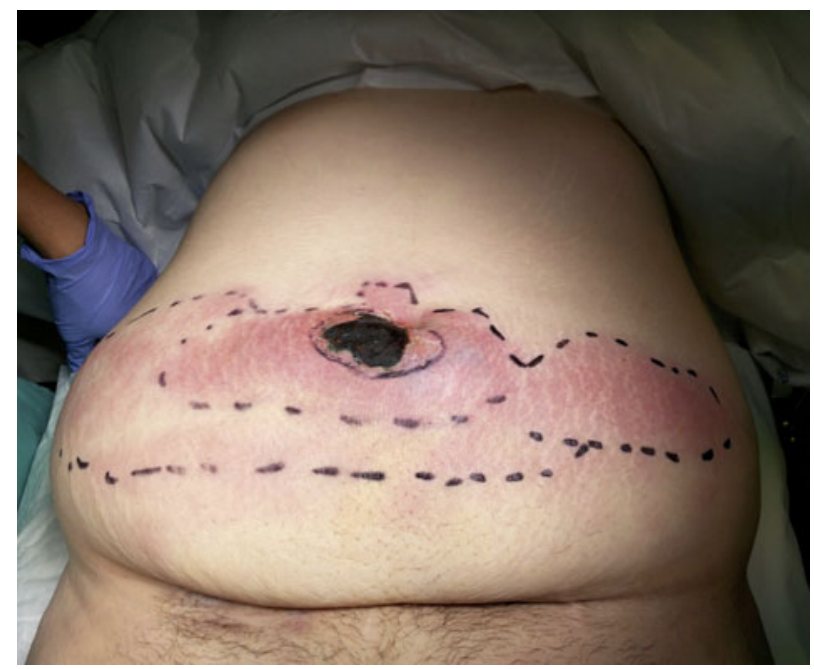

FIG. 1. A 3-cm diameter periumbilical necrotic eschar was found, surrounded by erythema spanning toward both flanks.

had never been treated. She came to the emergency department with general malaise and vomiting of 4 days' duration and a glycemia of $470 \mathrm{mg} / \mathrm{dL}$. Upon arrival, she was normotensive, tachycardic (116 beats per minute), and afebrile. At physical examination, a 3-cm diameter peri-umbilical necrotic scar was found, surrounded by erythema spanning toward both flanks (Fig. 1). The patient reported having detected the lesions 72 hours before admission.

The initial blood investigation revealed serum glucose concentration of $559 \mathrm{mg} / \mathrm{dL}$, elevated C-reactive protein (CRP; $38.3 \mathrm{mg} / \mathrm{dL})$, elevated lactic ( $2 \mathrm{mmol} / \mathrm{L})$, leucocytosis $\left(39.7 \times 10^{9} / \mathrm{L}\right)$, and neutrophilia $(85.2 \%)$ as well as dyselectrolytemia with hyponatremia $(123.2 \mathrm{mmol} / \mathrm{L})$, hypochloremia ( $96 \mathrm{mmol} / \mathrm{L})$, and hypokalemia $(3.07 \mathrm{mmol} / \mathrm{L})$. She was in metabolic acidosis with pHa of 7.2. A CT scan revealed multiple gas bubbles with fat stranding dissecting the anterior abdominal wall, without clear muscular involvement. It spread at least $31 \times 14 \times 8 \mathrm{~cm}$ and was compatible with necrotizing abdominal wall infection (Fig. 2). Piperacillin-tazobactam plus vancomycin were prescrived empirically. Urgent surgical

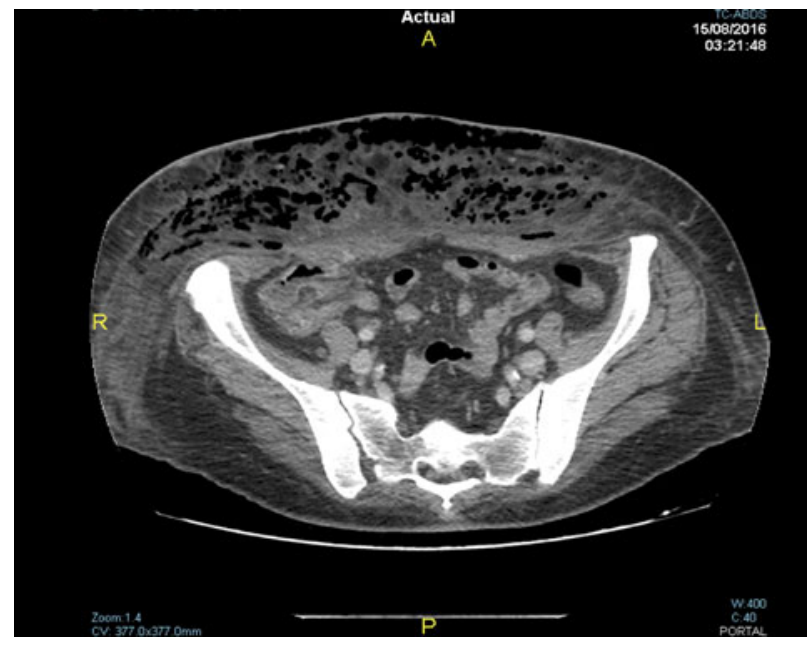

FIG. 2. Computed tomography compatible with necrotizing abdominal wall infection. 


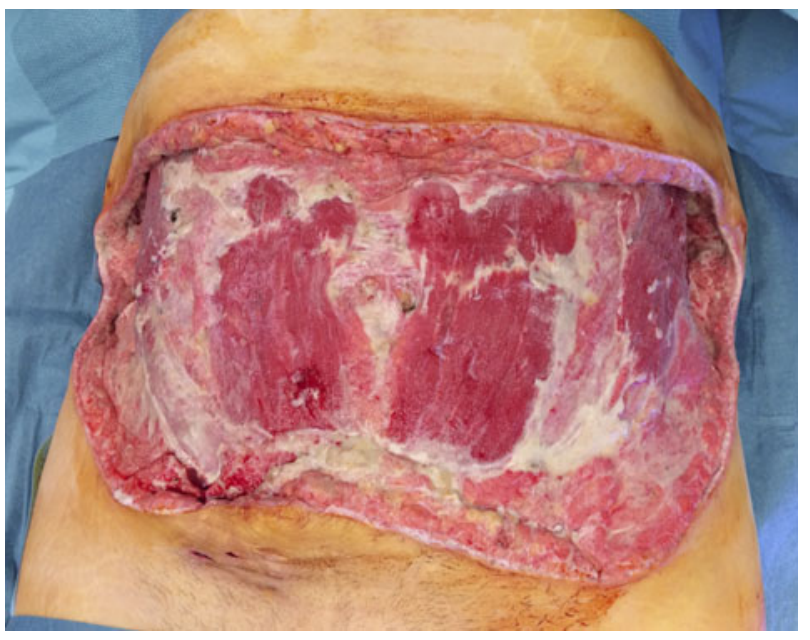

FIG. 3. Urgent debridement was carried out by means of a wide horizontal fusiform resection through non-affected areas.

debridement was performed by means of a wide horizontal fusiform resection through non-affected areas, extending from the navel to the xiphoid appendix and reaching both flanks removing the anterior fascia of the rectus abdominis muscle (Fig. 3). After profuse irrigation with saline, a negative pressure wound therapy (NPWT) system was applied. A second debridement was needed in the following 24 hours and then multiple replacement of NPWT for 26 days.

The wound exudate cultures grew Acinetobacter baumannii, Streptococcus agalactiae, Actimonyces funkey, and Klebsiella pneumoniae BLEE. Cotrimoxazole was added for 15 days. Subsequently, the surgical site exudate was positive for multi-resistant Pseudomonas aeruginosa and therefore amikacin and ceftazidime were prescribed for 12 days. The patient presented urinary tract infection with urine culture positive for Escherichia coli resistant to cotrimoxazole and ampicillin, as well as for Candida spp. After 62 days of treatment, the cultures were negative and abdominal wall reconstruction could be performed. A direct closure by abdo-

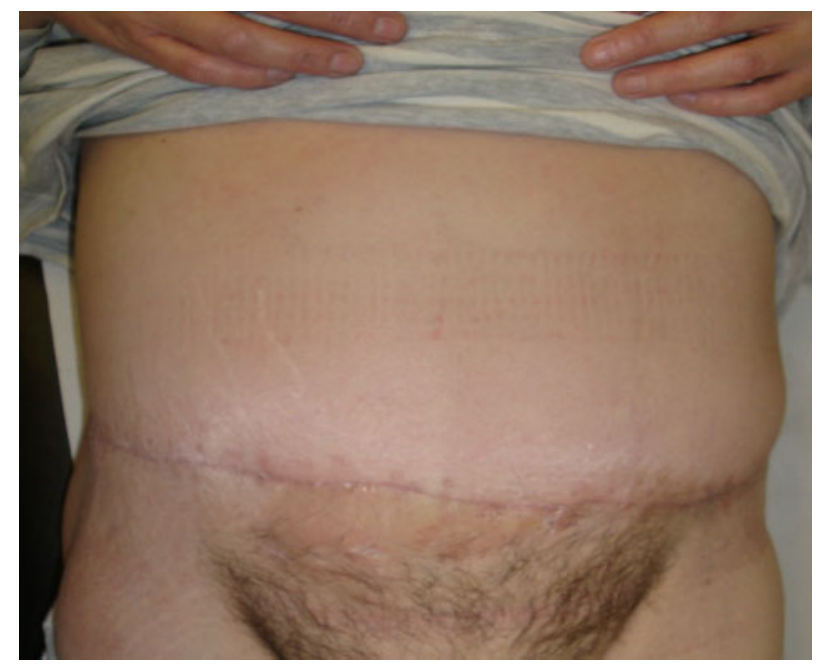

FIG. 4. Reconstruction using direct closure by abdominoplasty. minoplasty was performed in collaboration with the plastic surgery team, raising the upper abdominal skin flap, dissecting the lower pubic flap after removing the granulation tissue of the entire exposed abdominal wall (Fig. 4). In parallel, a strict glucose control was established with intravenous/subcutaneous insulin and the endocrinology department provided education and general diabetes mellitus management to the patient to facilitate transition to outpatient care and improve long-term glycemic control. The clinical condition of the patient improved without further complications and she could be discharged after 75 days of hospital admission.

\section{Discussion and Literature Review}

It has been reported that the majority of patients with NF have some associated immunodeficiency, diabetes mellitus being one of the most important pre-disposing conditions [9]. It plays an important role in the etiology of NF, not only by the underlying immunodeficiency, but also by other mechanisms such the tissue hypoxia caused by the diabetic vascular disease and the susceptibility to minor trauma caused by sensory polyneuropathy. Thus, clinicians must take diabetes mellitus as a red flag especially when dealing with suspicious clinical features.

Negative-pressure wound therapy can be considered because it has been shown to have favorable outcomes, most notably, the absence of additional wound necrosis, decrease in wound size, improvement of the wound quality, and quickening the recovery of the patient [10].

\section{Conclusion}

In conclusion, NF of the entire anterior abdominal wall is unusual and carries a poor prognosis if misdiagnosed at the initial stage as well as if an adequate management and early surgical treatment are not performed.

\section{Author Disclosure Statement}

No competing financial interests exist.

\section{References}

1. Sitges-Serra, A. (2007). Severe soft tissue infections: a syndrome-based approach. In Infectious Diseases in Critical Care (pp. 521-530). Springer Berlin Heidelberg.

2. Goh T, Goh LG, Ang CH, Wong CH. Early diagnosis of necrotizing fasciitis. Br J Surg 2014;101:e119-e125.

3. Bilton BD, Zibari GB, McMillan RW, et al. Aggressive surgical management of necrotizing fasciitis serves to decrease mortality: A retrospective study. Am Surg 1998;64: 397-400.

4. Giuliano A, Lewis F, Hadley $\mathrm{K}$, et al. Bacteriology of necrotizing fasciitis. Am J Surg 1977;134:52-57.

5. Stevens DL, Bisno AL, Chambers HF, et al. Practice guidelines for the diagnosis and management of skin and soft-tissue infections. Clin Infect Dis 2005;41:1373-1406.

6. Jones ME, Karlowsky JA, Draghi DC, et al. Epidemiology and antibiotic susceptibility of bacteria causing skin and soft tissue infections in the USA and Europe: A guide to appropriate antimicrobial therapy. Int J Antimicrob Agents 2003;22:406-419.

7. Wong $\mathrm{CH}$, Khin LW, Heng KS, et al. The LRINEC (Laboratory Risk Indicator foR Necrotizing Fasciitis) score: A tool for distinguishing necrotizing fasciitis from other soft tissue infections. Crit Care Med 2004;32:1535-1541. 
8. Yilmazlar T, Ozturk E, Alsoy A, et al. Necrotizing soft tissue infections: APACHE II score, dissemination, and survival. World J Surg 2007;31:1858-1862.

9. Cheng N-C, Tai H-C, Chang S-C, et al. Necrotizing fasciitis in patients with diabetes mellitus: clinical characteristics and risk factors for mortality. BMC Infect Dis 2015;15:417.

10. Lee JY, Jung H, Kwon H, et al. Extended negative pressure wound therapy-assisted dermatotraction for the closure of large open fasciotomy wounds in necrotizing faciitis patients. World J Emerg Surg 2014;9:29-39.

Address correspondence to: Dr. Juan Sancho-Insenser Digestive and General Surgery Department Hospital del Mar. Passeig Marítim 25-29 08003, Barcelona Spain

E-mail: jjsancho@gmail.com

$\begin{aligned} & \text { Abbrevition Used } \\ & \mathrm{NF}=\text { necrotizing fasciitis } \mathrm{CRP}=\mathrm{C} \text {-reactive protein } \\ & \mathrm{CT}=\text { computed tomography } \\ & \mathrm{NPWT}=\text { negative pressure wound therapy }\end{aligned}$

Cite this article as: Torrent-Jansà L, MembrillaFernández E, Climent-Agustín M, Silva-Vergara C, Martínez-Sola A, Toro-Araunabeña S, Pons-Fraguero MJ, Isbert-Trullàs FN, Guzman-Ahumada J, LópezFernández S, Grande-Posa L, Sancho-Insenser J (2017) Necrotizing abdominal fasciitis in un-treated diabetes mellitus. Surgical Infections Case Reports 2:1, 116-119, DOI: $10.1089 /$ crsi.2017.0030 